\title{
Letter
}

\section{e Patient Negativity as an Obstacle to the Treatment of Chronic Pain and a Role for Cognitive Bias Modification}

Omar Viswanath, MD¹, Nathaniel Beyer, MA, MAT², Ivan Urits, MD², and Alan David Kaye, MD, $\mathrm{PhD}^{3}$

From: ${ }^{1}$ Valley Anesthesiology and Pain Consultants, Phoenix, AZ; Department of Anesthesiology, University of Arizona College of Medicine, Phoenix, AZ; and Department of Anesthesiology, Creighton University School of Medicine, Omaha, NE; 2Department of Anesthesia, Critical Care, and Pain Medicine, Beth Israel Deaconess Medical Center, Harvard Medical School, Boston, MA; ${ }^{3}$ Department of Anesthesiology, Louisiana State University School of Medicine, New Orleans, LA

Address Correspondence: Omar Viswanath, MD Valley Anesthesiology and Pain Consultants

645 E. Missouri Ave., Suite 300 Phoenix, AZ 85012

E-mail: viswanoy@gmail.com

Disclaimer: There was no external funding in the preparation of this manuscript. Conflict of interest: Each author certifies that he or she, or a member of his or her immediate

family, has no commercial association (i.e., consultancies, stock ownership, equity interest, patent/licensing arrangements, etc.) that might pose a conflict of interest in connection with the submitted manuscript.

Manuscript received: 02-27-2019 Accepted for publication: 03-11-2019

Free full manuscript: www.painphysicianjournal.com
Various strategies have been reported to help physicians better manage challenging patient interactions and improve communication $(1,2)$. Furthermore, evidence suggests that rudeness among physicians has been demonstrated to negatively impact patient care $(3,4)$. Few investigators, however, have examined the impact that patient negativity, directed toward physician providers, may have on the quality of patient care. Although patient negativity may emerge in any care setting, it poses a particular challenge in the outpatient treatment of chronic pain, and thus, may influence the experience and treatment of this patient population.

Patient negativity stems from an interplay of past personal and environmental factors. Frequently, comorbid psycho-affective conditions including character disorder, depressive disorder, and anxiety disorder are intertwined with pain symptoms. Moreover, environmental factors, such as increased appointment wait times, paymentrelated barriers, travel for appointments, failed treatments, and perceived physician attitude may contribute to negative attitudes and behaviors. Over time, these factors may contribute to the provocation of outward negativity from patients. A failure to understand these symptoms, as well as the psychosocial aspects of pain treatment, can result in greater patient negativity and compromised treatment outcomes.

A growing literature of interest to pain physicians and staff are studies conducted in Israeli professional training settings among physicians and nurses (1). This work documents that patient rudeness in simulated settings can have deleterious effects on communication within and the performance of medical teams. Although relying on a simulation model, this research points to negative patient attitudes and verbal behaviors as important targets to evaluate and modify. Medical professionals in Israel and elsewhere are bringing to light the importance of studies in real-life contexts on the impact of patient negativity and modification strategies to combat unfortunate clinical outcome effects (5-7). These include techniques such as cognitive bias modification, which aims to directly change automatic cognitive processes, modify choice board, and thus, influence psychopathology (8).

Evaluation and management of patient negativity in the outpatient setting can contribute meaningfully to treatment planning and results. Many factors may lead patients to express negativity directed at their pain physician; this article highlights the adverse impact that such negative attitudes may have on patient care. Despite receiving training in communication-based tools to prevent underlying adverse effects on care, physicians may fail to recognize subconscious biases that may inherently compromise patient care. This brings to light the importance and need for more studies to assess the impact of patient negativity on quality of care, and to provide potential strategies that clinicians can be educated on to help mitigate this effect. Additionally, 
we need the implementation of this education skillset into our training programs as a foundation to combat deleterious responses toward our patients. Ultimately, it is imperative that chronic pain physicians be cognizant of internal patient factors such as prolonged failed therapy, comorbid conditions, and personal biases, which may serve as an impetus for patient frustration, manifesting as negativity, and thus, impeding positive outcomes. The propagation and dissemination of this knowledge to both physicians and patients can continue to help and strengthen the bond between both with the goal of improved and healthy communication with better overall quality of care.

\section{Acknowledgments}

The authors acknowledge Allan F. Simpao, M.D., M.B.I., Assistant Professor of Anesthesiology and Critical Care, Department of Anesthesiology and Critical Care Medicine, Children's Hospital of Philadelphia and the University of Pennsylvania Perelman School of Medicine, Philadelphia, PA, USA.

\section{REFERENCES}

1. Burnell RI. The right to be rude: managing conflict. Nurs Times 2016; 112:16-19.

2. Winner J. Reducing frustration and increasing fulfillment: Mindfulness. Fam Pract Manag 2017; 24:28-32.

3. Paice E, Smith D. Bullying of trainee doctors is a patient safety issue. Clin Teach 2009; 6:13-17.

4. Launer J. Rudeness and respect in medi- cine. Postgrad Med J 2016; 92:307-308.

5. Riskin A, Erez A, Foulk TA, Kugelman A, Gover A, Shoris I, Riskin KS, Bamberger PA. The Impact of rudeness on medical team performance: A randomized trial. Pediatrics 2015; 136:487-495.

6. Riskin A, Erez A, Foulk TA, Riskin-Geuz KS, Ziv A, Sela R, Pessach-Gelblum L, Bamberger PA. Rudeness and medi- cal team performance. Pediatrics 2017; 139:e20162305.

7. MacLeod C, Mathews A. Cognitive bias modification approaches to anxiety. Annu Rev Clin Psychol 2012; 8:189-217.

8. Jones EB, Sharpe L. Cognitive bias modification: A review of meta-analyses. J Affect Disord 2017; 223:175-183. 